\title{
The $g$ factor of conduction electrons in aluminium: calculation and application to spin resonance
}

\author{
F Beuneu \\ + Section d'Etude des Solides Irradiés. Centre d'Etudes Nucléaires. 92260 Fontenay-aux- \\ Roses. France \\ and \\ Laboratoire de Physique des Solides (Laboratoire associé au CNRS). Université Paris-Sud, \\ 91405 Orsay. France
}

Received 19 March 1980, in final form 30 May 1980

\begin{abstract}
We calculate the $y$ factor at every point of the Fermi surface of aluminium by using a classical four orthogonalised plane waves scheme and by introducing the spinorbit potential as a perturbation. An important difficulty remains. linked to the choice of the wavefunction phase. Moreover we propose a phenomenological model based on the narrowing of the $y$ distribution by two types of motion: a random one corresponding to the diffusion of electrons on the crystalline imperfections and a coherent one around the cyclotron orbits. A qualitative model accounts relatively well for the spin resonance experimental data.
\end{abstract}

\section{Introduction}

The conduction electron spin resonance (CESR) of aluminium has been studied extensively over the last few years. The main parameters of the resonance ( $g$ factor and linewidth) show a dependence with temperature and also with the frequency of the spectrometer which is not understood at present. In this paper we propose a phenomenological model accounting qualitatively for the experiments and based on a calculation of the $g$ factor of $\mathrm{Al}$ at every point of the Fermi surface.

In two previous papers (Beuneu and Monod 1978, Monod and Beuneu 1979) we compared the CESR properties of many metals. We pointed out that the linewidth due to phonons is anomalously broad for all the polyvalent metals, and we interpreted this fact as being due to inhomogeneous spin scattering of electrons depending on their position on the Fermi surface. Such an idea makes the detailed study of the behaviour of aluminium particularly attractive. because it can lead to more understanding of the CESR properties of many non-s (polyvalent) metals.

We summarise here the main results concerning the anomalous magnetic properties of aluminium. Static susceptibility measurements (Delafond et al 1973. Mimault et al 1973.) give some unexplained results: the static susceptibility is $6.3 \times 10^{-7} \mathrm{EMU} \mathrm{g}^{-1}$ at $290 \mathrm{~K}$, a value larger than the free-electron value for the Pauli susceptibility, namely $4.6 \times 10^{-7}$ EMU $^{-1}$. This experimental susceptibility decreases by some $30 \%$ between $10 \mathrm{~K}$ and $300 \mathrm{~K}$. The same authors report considerable variations of susceptibility with alloying. CESR experiments in aluminium show interesting features. The

+ Present address. 
variation of the linewidth with temperature has been studied at a number of different frequencies: $1.27 \mathrm{GHz}$ (Lubzens and Schultz 1976), 9.2 GHz (Lubzens et al 1972, Sambles et al 1977b), $21 \mathrm{GHz}$ (Janssens et al 1975), $35 \mathrm{GHz}$ (Lubzens et al 1972), $60 \mathrm{GHz}$ (Van Meijel et al 1977) and $79 \mathrm{GHz}$ (Dunifer and Pattison 1976). It appears from these experiments that:

(i) the linewidth is frequency dependent at all temperatures;

(ii) at a given temperature, the frequency dependence is linear, the slope decreasing by less than a factor of two from the low temperatures to $80 \mathrm{~K}$ (Sambles et al 1977a);

(iii) at frequencies larger than $10 \mathrm{GHz}$, the linewidth shows a minimum with temperature, between $20 \mathrm{~K}$ and $40 \mathrm{~K}$.

On the other hand, the $g$ factor varies with frequency and temperature in the $0-50 \mathrm{~K}$ range.

More precisely, at frequencies lower than $10 \mathrm{GHz}$ the $g$ value is frequency and temperature independent and is known to be $1.996 \pm 0.001$. At higher frequencies, the $g$ value is 1.996 near $50 \mathrm{~K}$ but increases with decreasing temperature, the increase being faster for larger frequencies.

We have to mention here that in all these experiments there are important effects due to surface relaxation which are not understood clearly yet. Surfaces can influence CESR data in two ways: firstly, the momentum relaxation introduces motional narrowing, and secondly, the spin relaxation gives extra broadening of the lines; the two competing mechanisms give experimental data which are difficult to analyse. For a first study of surface spin relaxation in aluminium, see Sharp-Dent et al (1976).

The linewidth data are generally believed to be due to a $g$ factor anisotropy over the aluminium Fermi surface. A motional narrowing mechanism would be responsible for the decrease of the linewidth with decreasing frequency and for the minimum of the linewidth with temperature at high frequencies. However, this model is unsatisfactory, as it cannot account for:

(i) the linear dependence of the linewidth with frequency (the simple motional narrowing model predicts a quadratic frequency dependence);

(ii) the small variation of the slope of this dependence with temperature (at high temperature, where the momentum collisions are very frequent, the $g$ factor distribution should be completely narrowed, contrasting with the experimentally important variation of linewidth with frequency up to $80 \mathrm{~K}$ ).

A more complicated model, based on many-body effects, has been proposed (Fredkin and Freedman 1972, Freedman and Fredkin 1975) but no agreement with the two points above has been obtained. From the linear frequency dependence of the linewidth, it has sometimes been proposed that there should be no motional narrowing at all in aluminium. However this hypothesis requires a scattering time of the order of $10^{-8} \mathrm{~s}$, even at $80 \mathrm{~K}$ where the resistivity scattering time is about $10^{-13} \mathrm{~s}$. This disagreement of five orders of magnitude makes nonsense of the non-motional narrowing model.

One may then consider the CESR properties of aluminium to be unexplainedt.

\footnotetext{
$\dagger$ Recent reports on the variations of CESR properties with frequency (in Ag and Na: Braim et al 1979; in Cu Stesmans et al 1979) seem to indicate an anomalous, aluminium-like behaviour of the linewidth with temperature and frequency. However the situation is not clear at present. as Dunifer (1980) gives results for copper which contradict those of Stesmans et al (1979). On the other hand, preliminary measurements on palladium metal (A Stesmans and $\mathbf{P}$ Monod. private communication) seem to be consistent with a very large frequency dependence of the linewidth.
} 
It is the purpose of the present paper to give a tentative model for these properties. Our model is based on a detailed calculation of the $g$ factor value at each point of the Fermi surface. $g$ factor calculations are not very numerous in the literature, and most of them deal with semiconductors (Roth 1960) or semimetals (Cohen and Blount 1960). For metals we can mention calculations in alkalis (Yafet 1957, 1963, Bienenstock and Brooks 1964, Moore 1975b), iron and nickel (Singh et al 1976) and palladium (Lenglart 1967, Rahman et al 1978). Except in the last paper cited, no effort has been made to relate the calculated $g$ factor distribution over the Fermi surface to the CESR linewidth. The only available model is that of Fredkin and Freedman (1972, see also Freedman and Fredkin 1975) which is only based on a phenomenological description of the $g$ factor distribution. In this paper we present a model for the motional narrowing in aluminium taking into account the calculated $g$ distribution. This phenomenological approach considers two types of motion: a random one, due to momentum scattering and a coherent one, the cyclotron motion.

\section{The wavefunction of conduction electrons in aluminium}

We use here a very simple method, due to Ashcroft (1963), for computing the Fermi surface of aluminium. The first step neglects the spin-orbit potential, this approximation being justified by the fact that the $3 p$ atomic splitting due to the spin-orbit is about $10^{-3}$ Ryd, a value much lower than the typical band gaps in the metal. The introduction of the spin-orbit potential is made using perturbation theory, and constitutes the second step of the present section.

Ashcroft's method uses four orthogonalised plane waves (OPW) as basis functions for the description of the first conduction bands in aluminium (four is the minimum number of OPW that one must consider in order to give a satisfactory description of the Brillouin zone corner, called $W$, which is the point needing the greatest number of OPW). The pseudopotential is introduced phenomenologically by fitting its first Fourier components in such a way as to obtain a satisfactory picture of the Fermi surface compared with the data obtained in the de Haas-van Alphen experiments and the resulting secular equation is given in $\$ 4$. At each point of the Fermi surface we compute:

(i) the wavefunction at the Fermi level, written as a linear (and normalised to unity) combination of the four opw;

(ii) the three other energies corresponding to the three other solutions of the secular equation:

(iii) the wavefunctions for these three energy bands.

When determining this bandstructure, one finds that the point which has the coordinates $k_{x}=0.9649, k_{y}=0.4115$ and $k_{z}=0$ (in $2 \pi / a$ units) is quite peculiar: at this point of the Fermi surface, two energy bands are degenerate; in other words the second and third zone of the Fermi surface are in contact. This degeneracy will be lifted only by the spin-orbit and the treatment of this peculiar point is given in the appendix.

We arrive now at the point where spin-orbit perturbation must be considered. This interaction is written under the hypothesis that $V(r)$, the one-electron potential, is 
spherically symmetric:

$$
V_{\mathrm{so}}=\frac{\hbar^{2}}{4 m^{2} c^{2}} \frac{1}{r} \frac{\partial V}{\partial r} l . s=\lambda(r) l . s=\lambda(r)\left[l_{z} s_{z}+\frac{1}{2}\left(l_{+} s_{-}+l_{-} s_{+}\right)\right]
$$

where $z$ is the quantisation axis and

$$
l_{ \pm}=l_{x} \pm \mathrm{i} l_{y} \quad s_{ \pm}=s_{x} \pm \mathrm{i} s_{y} .
$$

We wish to determine how the wavefunction $\psi_{i}$ (of wavevector $\boldsymbol{k}$ ) is modified with the introduction of the spin-orbit. $V_{\text {so }}$ couples $\psi_{i}$ with the other wavefunctions of the same $\boldsymbol{k}$, which we denote by $\psi_{j}$. Here we shall consider only the three other conduction wavefunctions obtained above in the four OPw method as $\psi_{j}$; we neglect the influence of the bands of higher order as well as the underlying levels, this being justified in a perturbation treatment by the large energy denominators linked to these bands. Following Elliott (1954) we write the wavefunction, as modified in first order in the spin-orbit perturbation, as follows:

$$
\hat{\psi}_{i}=\psi_{i}|+\rangle+\frac{1}{2} \sum_{j \neq i}\left(\frac{\left\langle\psi_{j}\left|\lambda(r) l_{z}\right| \psi_{i}\right\rangle \psi_{j}}{E_{i}-E_{j}}|+\rangle+\frac{\left\langle\psi_{j}\left|\lambda(r)\left(l_{x}+i l_{y}\right)\right| \psi_{i}\right\rangle \psi_{j}}{E_{i}-E_{j}}|-\rangle\right)
$$

where the symbol over $\hat{\psi}_{i}$ denotes that this function includes the spin-orbit contribution. The form for $\hat{\psi}_{i}$ is no longer correct when a band $j$ exists such that $\left|E_{i}-E_{j}\right|$ is of the order of the $3 \mathrm{p}$ spin-orbit splitting $\left(\approx 10^{-3} \mathrm{Ryd}\right)$. This is the case only in the immediate neighbourhood of the point of degeneracy already mentioned (see the appendix).

We have to determine the matrix elements of the spin-orbit between two opw. Let us write:

$$
\left|\mathrm{OPW}_{i}\right\rangle=(1-P)\left|k_{i}\right\rangle=\left|k_{i}\right\rangle-\sum_{x}|\alpha\rangle\left\langle\alpha \mid k_{i}\right\rangle
$$

where the $\alpha$ are taken to be the $1 \mathrm{~s}, 2 \mathrm{~s}$ and $2 \mathrm{p}$ states of the $\mathrm{Al}^{3+}$ ion. Using the fact that $l_{z}$ and $(1-P)$ commute, we get:

$$
\begin{aligned}
\left\langle\mathrm{OPW}_{j}\left|\hat{l_{z}}\right| \mathrm{OPW}_{i}\right\rangle & =\left\langle k_{j}\left|\lambda l_{z}(1-P)\right| k_{i}\right\rangle \\
& =\left\langle k_{j}\left|\lambda l_{z}\right| k_{i}\right\rangle-\sum_{x}\left\langle k_{j}\left|\lambda_{z}\right| \alpha\right\rangle\left\langle\alpha \mid k_{i}\right\rangle .
\end{aligned}
$$

The first term of the last expression is very small. This is rather intuitive because the spin-orbit acts principally on the core part of the wavefunction. This term will be neglected.

Writing:

$$
x_{n l m}=\frac{P_{n l}(r)}{r} Y_{l}^{m}(\theta, \phi)
$$

where $n, l$ and $m$ are the classical quantum numbers associated with the core state $\alpha$, and defining

$$
i_{n l}=\int_{0}^{x} \lambda(r) P_{n l}^{2}(r) \mathrm{d} r
$$


we can immediately deduce the following equation from the fact that the $\alpha$ are a basis of eigenfunctions for the $\lambda l_{z}$ operator:

$$
\left\langle k_{j}\left|\lambda_{z}\right| \alpha_{n l m}\right\rangle=m i_{m l}\left\langle k_{j} \mid \alpha_{n l m}\right\rangle .
$$

In the case of $\mathrm{Al}^{3+}$, the only $x$ to be considered, i.e. of non-zero $m$, are the $2 \mathrm{p}$ states of $m= \pm 1$. The $i_{2 \mathrm{p}}$ parameter will be chosen from the data corresponding to the atomic spin-orbit splittings as tabulated by Yafet (1963).

The preceding treatment of the $\lambda l_{z}$ matrix element is very similar to the one made by Asik et al (1969).

We have to compute the scalar products $\langle\alpha \mid k\rangle$ between the $2 p$ core states and the plane waves. The calculation is done easily (see for instance Harrison 1966) by expanding the plane wave in terms of spherical harmonics and using orthogonalisation of these spherical harmonics. We obtain

$$
\langle\alpha \mid k\rangle=\frac{4 \pi}{\Omega_{0}^{1 / 2}} \mathrm{i}^{l} Y_{l}^{m *}\left(\theta_{k}, \phi_{k}\right) \int_{0}^{\infty} j_{l}(k r) r P_{n l}(r) \mathrm{d} r
$$

where $\Omega_{0}$ is the volume of the unit cell of the crystal.

Defining

$$
I(k)=\int_{0}^{x} j_{1}(k r) r P_{2 p}(r) \mathrm{d} r
$$

we obtain by a straightforward calculation:

$$
\left\langle\mathrm{OPW}_{j}\left|\lambda_{z}\right| \mathrm{OPW}_{i}\right\rangle=\mathrm{i} \lambda_{2 \mathrm{p}} \frac{12 \pi}{\Omega_{0}} \frac{I\left(k_{i}\right)}{k_{i}} \frac{I\left(k_{j}\right)}{k_{j}}\left(k_{y}^{i} k_{x}^{j}-k_{y}^{j} k_{x}^{i}\right) .
$$

The $I(k)$ integral is calculated numerically using the values of Hartree (1935) for $P_{2 \mathrm{p}}(r)$ in $\mathrm{Al}^{3+}$. Values of $I(k)$ are tabulated by Beuneu (1979).

\section{Calculation of the $g$ factor}

The problem of the Bloch electrons in a magnetic field is known to be a very complicated one: a field, even a small one, does not have a small effect on the wavefunctions because they extend to infinity throughout the crystal, and thus perturbation theory cannot be used. This difficult theoretical problem has been solved by Kohn (1959), Roth (1962) and Blount (1962). The general idea for the treatment of the magnetic field is that if the change in the wavefunctions is large, the change in the energy levels is small for ordinary fields, so that one can obtain an effective Hamiltonian where bands are decoupled.

Yafet (1963) shows in detail how such a method can be used to obtain $g$ factors in crystals, following the work of Cohen and Blount (1960) for bismuth.

As a first step, consider the case of a band edge: the problem is somewhat simpler and it applies directly to semiconductors. It is useful to define a new operator, called $\boldsymbol{X}$, which behaves as the periodic part of the position operator $\boldsymbol{x}$. Consider two Bloch 
waves $\Psi_{n}$ and $\Psi_{n}$, with periodic parts $u_{n}$ and $u_{n}$. By definition we write:

$$
X_{n n^{\prime}}^{x}=\frac{(2 \pi)^{3}}{\Omega_{0}} \int_{\text {cell }} u_{n}^{*} \frac{\mathrm{i} \hat{c} u_{n^{\prime}}}{\hat{c} k_{x}} \mathrm{~d} \tau
$$

where the integral is computed over the elementary cell of the crystal. The total magnetic moment can be given by

$$
\boldsymbol{\mu}=-\mu_{\mathrm{B}}\left(\boldsymbol{L}+\frac{1}{2} g_{0} \boldsymbol{\sigma}\right)
$$

where $g_{0}=2.0023$ is the free-electron $g$ value. The operator $L$ is defined by

$$
L=\hbar^{-1} X \times \pi
$$

where $\pi$ is given by

$$
\pi=p+\frac{\hbar}{4 m c^{2}} \sigma \times \nabla V .
$$

$\boldsymbol{L}$ can be interpreted as the periodic part of the angular momentum $\boldsymbol{l}: \boldsymbol{X}$ being a periodic operator, its matrix elements between Bloch functions converge, and the same thing is true for $\boldsymbol{L}$. On the contrary, neither $x$ nor $l$ have this property (this is clearly linked to the fact that the magnetic field cannot be treated by perturbation theory). Yafet (1957) showed that the difference between the matrix elements of $X$ and those of $\boldsymbol{x}$, taken over the unit cell between two Bloch functions, can be written as an integral over the surface of the unit cell; thus, if one moves the atoms of the crystal away from one another, $L$ reduces to $l$ in the limit of infinite interatomic distance.

With these definitions, the $g$ factor at a band edge is shown to be

$$
g=2\left\langle\psi \uparrow\left|L_{z}+2 s_{z}\right| \psi \uparrow\right\rangle
$$

with the condition that the matrix element of $L_{z}+2 s_{z}$ between $\psi \uparrow$ and $\psi \downarrow$ must be zero (or negligible). Consider now a solid where the spin-orbit is small: to first order in the spin-orbit the $s_{z}$ operator gives no deviation to $g=2$, so that if we define $\Delta g$ as $g-g_{0}$ we can write

$$
\Delta g=2\left\langle\psi \uparrow\left|L_{z}\right| \psi \uparrow\right\rangle .
$$

It must not be forgotten that such a formula, which neglects the non-diagonal terms in the magnetic (Zeeman) Hamiltonian, will give correct results only for $|\Delta g| \ll 2$. When the $\Delta g$ is calculated at band $i$, it is classical to write

$$
\Delta g_{i}=\frac{2}{\hbar} \sum_{j \neq i}\left(\hat{X}_{i j}^{x} \hat{\pi}_{j i}^{y}-\hat{X}_{i j}^{y} \hat{\pi}_{j i}^{x}\right)
$$

where $\hat{X}_{i j}^{x}$ is a condensed form of $\left\langle\hat{\psi}_{i}\left|X^{x}\right| \hat{\psi}_{j}\right\rangle$.

The $j$ index describes all the energy bands with the same wavevector, and ' $j \neq i$ ' in the sum because $\left\langle\hat{\psi}_{i}\left|\pi^{x}\right| \hat{\psi}_{i}\right\rangle$ is known to be zero at a band edge.

We are now at the point when we have to consider the second step; namely, the case of the Fermi surface electrons which are not at a band edge in the metal. It is interesting to show qualitatively why this case is more complicated than the preceding one. Using a semiclassical picture, we can say that the electron velocity is zero at a band edge; on the contrary, away from a band edge, the electron has a velocity $v$; this 
means that in a magnetic field $\boldsymbol{H}$ the electron has a cyclotron motion such that

$$
v=\omega \times x=\frac{e \boldsymbol{H}}{m c} \times \boldsymbol{x}
$$

which adds another contribution to the orbital magnetic momentum. The quantity $\boldsymbol{X}_{i i}(\boldsymbol{k})$ represents a displacement of the electronic charge of a wave-packet centred in $\boldsymbol{k}$; the magnetic moment linked to this displacement may be written as a vectorial product of $\boldsymbol{X}_{i i}$ with the electron velocity $\pi_{i i}$.

Blount (1962) and Roth (1962) have separately shown that the $L$ contribution to the magnetic moment can be written

$$
\left(\mu_{l}\right)_{i i}=-\mu_{\mathrm{B}} h^{-1}\left[(\boldsymbol{X} \times \pi)_{i i}+X_{i i} \times \pi_{i i}\right]
$$

which agrees with the qualitative argument given above.

This leads to the generalisation of the $\Delta g$ formula given in the case of the band edge. One obtains

$$
\Delta g=\frac{2}{\hbar} \sum_{j \neq i}\left(\hat{X}_{i j}^{x} \hat{\pi}_{j i}^{y}-\hat{X}_{i j}^{y} \hat{\pi}_{j i}^{x}\right)+\frac{4}{h}\left(\hat{X}_{i i}^{x} \hat{\pi}_{i i}^{y}-\hat{X}_{i i}^{y} \hat{\pi}_{i i}^{x}\right) .
$$

There is no a priori reason why one of the two terms of this sum should be dominant. Our computations in aluminium, in general, give contributions of the same order of magnitude. For a detailed comment on the possible appearance of very large $g$ factor values by using such formulae, see appendix B of the paper by Yafet (1963).

There are some important remarks which concern the $\Delta g$ formula. A most surprising one is that this $\Delta g$ is not unique; this is because the diagonal matrix element $\hat{X}_{i i}^{x}$ depends on the (arbitrary) phase of the wavefunction $\hat{\psi}_{i}$. This is clearly seen from the definition of $\hat{X}_{i i}^{x}$ :

$$
\hat{X}_{i i}^{x}=\frac{(2 \pi)^{3}}{\Omega_{0}} \int_{\text {cell }} \hat{u}_{i}^{*} \frac{\mathrm{i} \partial \hat{u}_{i}}{\partial k_{x}} \mathrm{~d} \tau .
$$

If one changes the phase of $\hat{\psi}_{i}$ by a factor depending on $k$, one adds to $\boldsymbol{X}_{i i}$ a quantity proportional to the gradient (in $k$ ) of this factor. Thus $\hat{X}_{i i}^{x}$, and consequently $\Delta g_{i}$, are not uniquely defined. Roth (1962) and Yafet (1963) partially solved this difficulty by showing that the mean $\Delta g$ over a cyclotron orbit is perfectly well defined (just because one has to integrate a gradient over a closed loop). However de Graaf and Overhauser (1969) remark that this argument is no longer satisfactory in the presence of scattering over the Fermi surface. As CESR can be observed in metal even when $\omega \tau \ll 1$ (for instance in molten $\mathrm{Na}$ ) they deduce that $g(\boldsymbol{k})$ must be well defined. We think that this statement is much exaggerated and does not constitute any proof (see $\$ 5$ ) but it is certain that an important and unresolved problem still remains.

As a second point, note that we shall use the approximation $\pi \simeq p$, as many authors do. In particular Roth (1962) justified this by showing that the contribution of the much neglected term to the $\Delta g$ is of the order of $\left\langle p^{2} / 2 m\right\rangle / m c^{2}=\left\langle v^{2}\right\rangle / c^{2} \ll 1$. With this approximation, we shall have no difficulty in computing the matrix elements of $\pi$ between linear combinations of the opw.

The determination of the matrix elements of the $X$ operator is as follows. The non-diagonal matrix elements are obtained by the formula:

$$
\boldsymbol{X}_{i j}=-\frac{i \hbar}{m} \frac{\pi_{i j}}{E_{i}-E_{j}} \quad \text { where } \quad i \neq j .
$$


This formula is demonstrated, in the zero spin-orbit case, by Wilson (1953) and the extension to the non-zero spin-orbit case is straightforward. The diagonal matrix elements are ambiguous, as discussed above, and we have to make an arbitrary choice for the phase of $\hat{\psi}_{i}$. For computational simplicity, we preferred to choose $\psi_{i}$, the wavefunctions without the spin-orbit. The explicit relations obtained will be given later.

We have given above the expression of the $g$ function relative to the wavefunction $\left|\hat{\psi}_{i}\right\rangle$, with quantisation along the $z$ axis. Here we write $\Delta g=\Delta g_{1}+\Delta g_{2}$ and compute the two terms separately. The above relation between $\boldsymbol{X}$ and $\pi$ leads to

$$
\Delta g_{1}=-\frac{2 \mathrm{i}}{m} \sum_{j \neq i} \frac{\hat{\pi}_{i j}^{x} \hat{\pi}_{j i}^{y}-\hat{\pi}_{i j}^{y} \hat{\pi}_{j i}^{x}}{E_{i}-E_{j}}
$$

which we can simplify by making the approximation $\pi \simeq p$. We must now write the $\hat{p}_{i j}$ as combinations of the $p_{i j}$; that is to say we introduce the spin-orbit terms explicitly. This is done by writing

$$
\left|\hat{\psi}_{i}\right\rangle=\left(\left|\psi_{i}\right\rangle+\mathrm{i} \sum_{m \neq i} i_{m}^{i}\left|\psi_{m}\right\rangle\right)|+\rangle+\left|c_{i}\right\rangle|-\rangle
$$

The term $\left|c_{i}\right\rangle$, to first order in the $i$ spin-orbit, was given explicitly above. Its effect on the $g$ shift is of second order and will be disregarded here. The term $\lambda_{m}^{i}$ is written

$$
\lambda_{m}^{i}=\frac{1}{2 \mathrm{i}} \frac{\left\langle\psi_{m}\left|\lambda l_{z}\right| \psi_{i}\right\rangle}{E_{i}-E_{m}}
$$

and is a real quantity. To first order in $\lambda$ one has

$$
\hat{p}_{i j}^{x}=p_{i j}^{x}+\mathrm{i} \sum_{n \neq j} i_{n}^{j} p_{i n}^{x}-i \sum_{m \neq i} \lambda_{m}^{i} p_{m j}^{x}
$$

It is easy to show (Beuneu 1979) that $p_{i j}^{x}$ is real.

With a few manipulations we can write the expression for $\Delta g_{1}$ as

$$
\Delta g_{1}=\frac{4}{m} \sum_{j \neq i} \frac{1}{E_{i}-E_{j}}\left(\sum_{n \neq j} \lambda_{n}^{j} p_{i n}^{x} p_{j i}^{y}+\sum_{m \neq i} \lambda_{m}^{i} p_{j i}^{x} p_{m j}^{y}-x \rightleftarrows y\right)
$$

where ' $x \rightleftarrows y$ ' symbolises the term obtained from the one explicitly found by permuting $x$ and $y$. The last formula can easily be shown to be the one obtained by Roth (1960) using a similar treatment though she needed no term like our $\Delta g_{2}$ as her calculation was performed at a band edge for the cases of silicon and germanium.

Consider now the term $\Delta g_{2}$ :

$$
\Delta g_{2}=\frac{4}{\hbar} \hat{X}_{i i}^{x} \hat{\pi}_{i i}^{y}-x \rightleftarrows y
$$

Although it is not true to write:

$$
X_{x}\left|\hat{\psi}_{i}\right\rangle=X_{x}\left|\psi_{i}\right\rangle+\mathrm{i} \sum_{m \neq i} i_{m}^{i} X_{x}\left|\psi_{m}\right\rangle
$$


because the $X$ operator must be applied to $\lambda_{m}^{i}$ as well, which is $\boldsymbol{k}$ dependent, the following formula can be written, always to first order:

$$
\hat{X}_{i i}^{x}=X_{i i}^{x}+\mathrm{i} \sum_{m \neq i} \lambda_{m}^{i}\left(X_{i m}^{x}-X_{m i}^{x}\right)
$$

This formula is true because $X$ gives only quantities which are not $x$-dependent with the $i_{m}^{i}$ term; it gives no extra contributions to the $x$ integrals calculated when determining $\hat{X}_{i i}^{x}$.

$X_{i m}^{x}$ is given by the formula

$$
X_{i m}^{x}=-\frac{i \hbar}{m} \frac{p_{i m}^{x}}{E_{i}-E_{m}}
$$

and thus is imaginary. The $X_{i i}^{x}$ term is determined only when we make an arbitrary choice for the phase of $\psi_{i}$. Following Roth (1962), we impose that $u_{i}(r=0)$ is real, which leads to

$$
X_{i i}^{x}=-\operatorname{Re} \sum_{m \neq i} \frac{u_{m}(0)}{u_{i}(0)} X_{m i}^{x} \quad \text { if } u_{i}(0) \neq 0 .
$$

In our case, we have just mentioned that $X_{m i}^{x}$ is imaginary for $m \neq i$; the $u_{m}(0)$ and $u_{i}(0)$ terms being real by our imposed condition, we obtain $X_{i i}^{x}=0$. The hypothesis $u_{i}(0) \neq 0$ does not seem restrictive. For a point where $u_{i}(0)=0$, the property $X_{i i}^{x}=0$ will be shown to be true by passing to the limit on a $k$ space path where $u_{i}(0) \neq 0$. Thus we have

$$
\hat{X}_{i i}^{x}=2 \mathrm{i} \sum_{m \neq i} i_{m}^{i} X_{i m}^{x}=\frac{2 \hbar}{m} \sum_{m \neq i} i_{m}^{i} \frac{p_{i m}^{x}}{E_{i}-E_{m}} .
$$

This expression carries no term of zero order in the spin-orbit.

In our first-order calculation, this enables us to replace $\hat{\pi}_{i i}^{y}$ by $p_{i i}^{y}$, its zero-order estimate, giving:

$$
\Delta g_{2}=\frac{8}{m} \sum_{m \neq i} \lambda_{m}^{i} \frac{p_{i m}^{x} p_{i i}^{y}}{E_{i}-E_{m}}-x \rightleftarrows y .
$$

We must not forget that one major difference between $\Delta g_{1}$ and $\Delta g_{2}$ is that the latter is somewhat arbitrary as its exact values depend on the choice of the phase, whereas $\Delta g_{1}$ is defined unambiguously.

\section{Numerical calculation and results}

The machine calculation was sufficiently simple to be performed on a desk-top calculator: the Hewlett-Packard $9825 \mathrm{~A}$. In the classical $1 / 48$ th of the Brillouin zone, we have studied some 8000 points with maximum density at the places where the Fermi surface curvature is high. This Fermi surface was determined by resolving Ashcroft's equation in its reduced shape (Ashcroft 1963), taking $2 \pi / a=1$ and $\hbar^{2} / 2 m=1$ and defining $T_{i}(\boldsymbol{k}, E)=\left(\boldsymbol{k}-\boldsymbol{g}_{i}\right)^{2}-E$ :

$\left(T_{0} T_{3}-V_{200}^{2}\right)\left(T_{1} T_{2}-V_{200}^{2}\right)-V_{111}^{2}\left(T_{0}+T_{3}-2 V_{200}\right)\left(T_{1}+T_{2}-2 V_{200}\right)=0$ 
with $\boldsymbol{g}_{0}=0 ; \boldsymbol{g}_{1}=(1,1,1) ; \boldsymbol{g}_{2}=(1,1,-1) ; \boldsymbol{g}_{3}=(2,0,0)$ and taking Ashcroft's values (in Ryd) of $E_{\mathrm{F}}=0.85605 ; V_{111}=0.0179$ and $V_{200}=0.0562$.

The matrix elements for the spin-orbit were computed by using the formula given in $\$ 2$, with the atomic value for $\lambda_{2 p}: \hat{\lambda}_{2 p}=0.27 \mathrm{eV}=0.0196 \mathrm{Ryd}$.

To determine the matrix elements of $p$, one has to calculate only the matrix elements between two OPW. It is reasonable to take

$$
\left\langle\mathrm{OPW}_{m}\left|p_{x}\right| \mathrm{OPW}_{n}\right\rangle=\hbar k_{x}^{m} \delta_{m n}
$$

which is true in an effective mass model. Such a model is good, as the large $\Delta g$ we shall obtain are due to the magnetism of intercellular currents circulating at large distances, and not to contributions inside one elementary cell for which the detail of the wavefunction's core oscillations should be considered.

Thus everything is determined in the formulae giving the values of $\Delta g_{1}$ and $\Delta g_{2}$. The only thing to be specified is the direction of the quantisation axis. here labelled the $z$ axis. We made the computations for the three simple orientations of the (100) type. It is interesting at this stage to show this theorem: at each point of the Brillouin zone, the sum of the $\Delta g$ obtained for three orthogonal axes of quantisation does not depend on the choice of these axes. Consider the formulae giving $\Delta g_{1}$ and $\Delta g_{2}$. They depend on the choice of the quantisation in two ways; the first is the spin-orbit matrix elements (given in $\$ 2$ ) and the second is the terms such as $p_{i n}^{x} p_{j i}^{y}-x \rightleftarrows y$. The two types of term depend finally in the same manner on the choice of axes, through a term like $\left(\boldsymbol{k}_{i} \times \boldsymbol{k}_{j}\right)_{z}$. Let $S=\Delta g_{x}+\Delta g_{y}+\Delta g_{z}$ be the sum of the $\Delta g$ with the quantisation axis along $x, y$ and $z$. S is proportional to

$$
\sum_{3 \text { axes }}\left(k_{i} \times k_{j}\right)_{z}\left(k_{m} \times k_{n}\right)_{z}=\left(k_{i} \times k_{j}\right) \cdot\left(k_{m} \times k_{n}\right)
$$

which is a scalar product of vectors and thus independent of the axes chosen; the theorem is proved. This theorem has an important consequence: in a motional narrowing regime for which the observed $\Delta g$ must be the mean of all the $\Delta g$ around the Fermi surface, it cannot depend on the orientation of the magnetic field relative to the crystal. This is no longer the case in the event of an absence of motional narrowing.

As we wish to obtain some means of $\Delta g$ we must determine the relative weight of each point of the Fermi surface. This weight must clearly be proportional to the density of states at the point $\boldsymbol{k}$ considered. If we write the four oPw secular equation of Ashcroft as $f(\boldsymbol{k}, E)=0$, the density of states is given by

$$
\frac{1}{\left|\nabla_{k} E\right|}=\frac{|\partial f / \hat{\partial} E|}{\left|\nabla_{k} f\right|}
$$

which we estimate numerically.

The numerical results for $\Delta g$ were given elsewhere (Beuneu 1979) in the form of long tables. We prefer to give a more 'visual' presentation of our results.

In figures 1-6, we show maps of the $\Delta g$ values on the Fermi surface. Figures 1-3 describe the second-zone points, and figures 4-6 the third-zone points. The projection procedure used here consists of defining two numbers, called $U$ and $T$, such that $k_{y}=U k_{x}$ and $k_{z}=T k_{x}$. The restriction to $1 / 48$ th of the zone leads to the inequalities $0 \leqslant T \leqslant 1$ and $T \leqslant U \leqslant 1$. These two numbers are the coordinates of the planar representations shown in figures $1-6$. To be clearer, let us note that this mode of projection is obtained by taking the intersection of the vector radius $\Gamma \mathrm{M}$ ( $\Gamma$ being the 


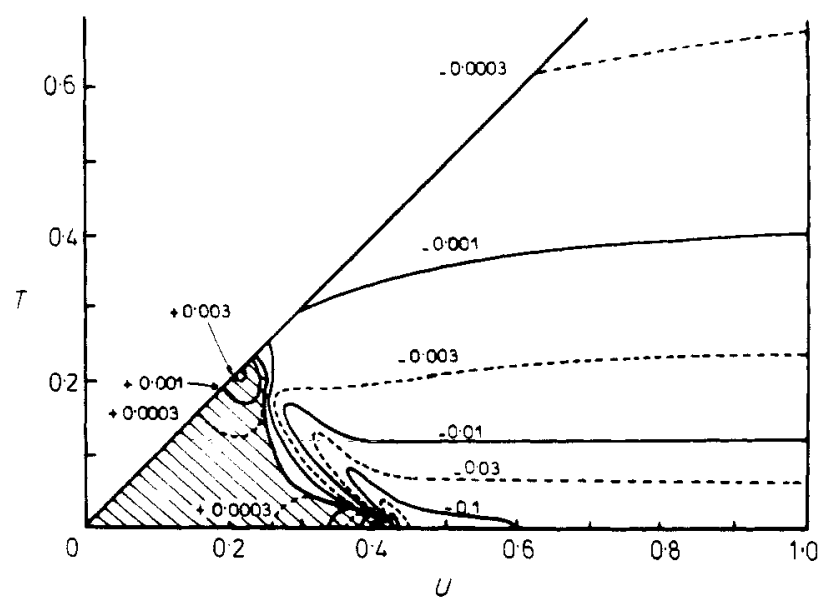

Figure 1. Reduced map of $\Delta g$ in aluminium for second-zone conduction electrons at the Fermi level, in the $1 / 48$ th of the zone chosen so that $k_{x} \geqslant k_{y} \geqslant k_{z} \geqslant 0$. The coordinates $U$ and $T$ are described in the text. The magnetic field axis is taken along $k_{x}$. The shaded part of the map corresponds to the positive $\Delta g$. Note the singular point at $U=0.4265$ and $T=0$ discussed in the appendix.

centre of the Brillouin zone and $\mathrm{M}$ the Fermi surface point we want to represent) with the $k_{x}=1$ plane where the map is drawn. This mode of projection enables us to obtain (nearly everywhere) two distinct couples $(U, T)$ for two distinct points on the Fermi surface taken in the same zone. For each zone, the three maps correspond to the three principal orientations of the magnetic field.

Due to the peculiar topology of the two- and three-zone volumes of the Fermi surface of aluminium, one can see that all the values of $U$ and $T$ correspond to a point in the second zone, and that this is not so for the 'arms' of the third zone. One property must be satisfied a priori by the maps: for symmetry reasons, the points in the $k_{x}=k_{y}$ plane must verify the relation $\Delta g_{x}=\Delta g_{y}$ (where the index, $x$ or $y$, indicates

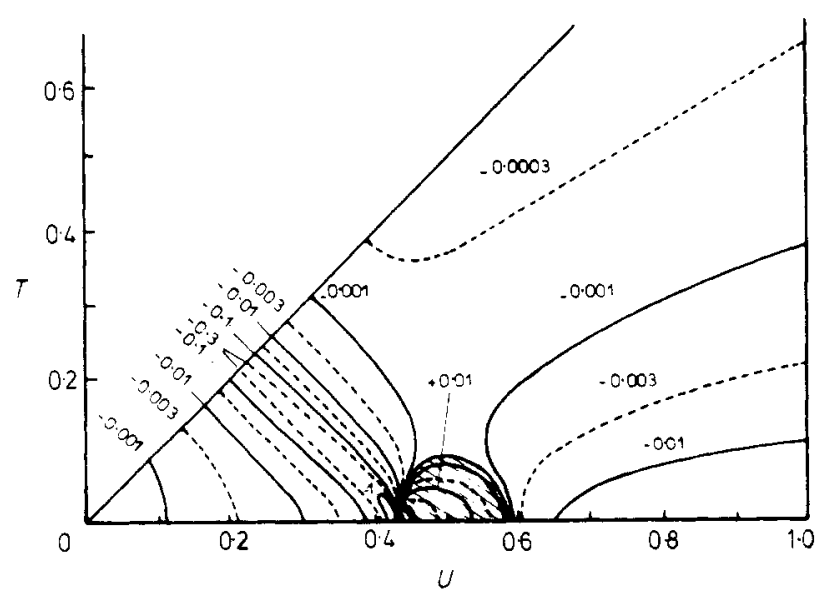

Figure 2. Reduced map of $\Delta g$ in aluminium for second-zone electrons. The magnetic field axis is along $k_{y}$. The coordinates $U$ and $T$ are the same as for figure 1 and the projection scheme is explained in the text. 


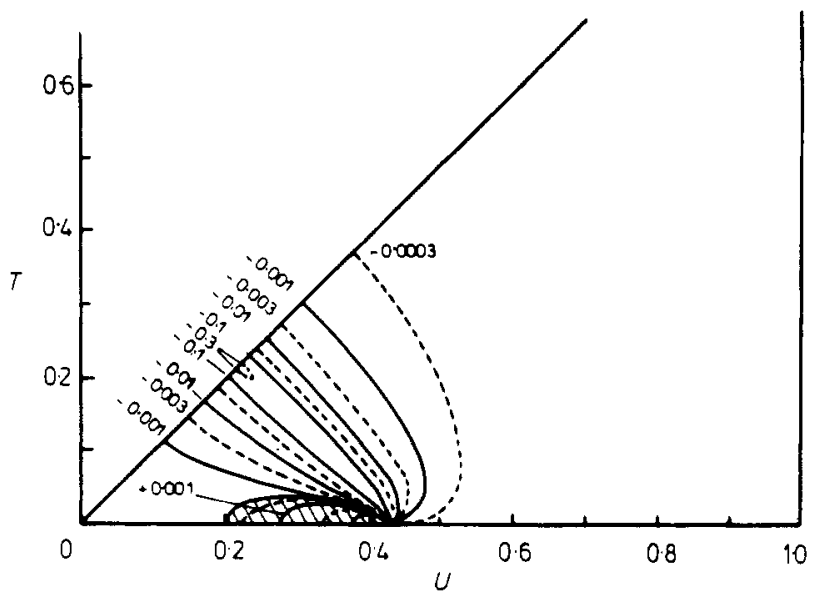

Figure 3. Reduced map of $\Delta g$ in aluminium for second-zone electrons. The magnetic field axis is along $k_{z}$.

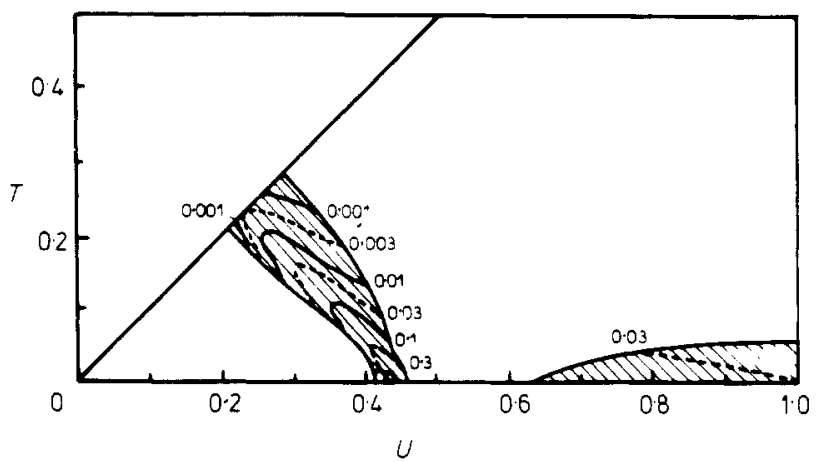

Figure 4. Reduced map of $\Delta g$ in aluminium for third-zone electrons. Note that not all the couples $(U, T)$ correspond to a third-zone point. The magnetic field axis is taken along $k_{x}$.

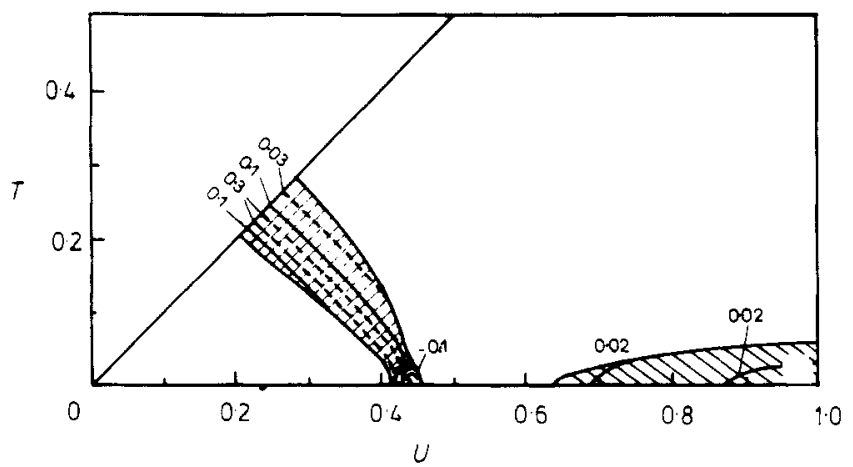

Figure 5. Reduced map of $\Delta g$ in aluminium for third-zone electrons. The magnetic field axis is along $k_{y}$ 


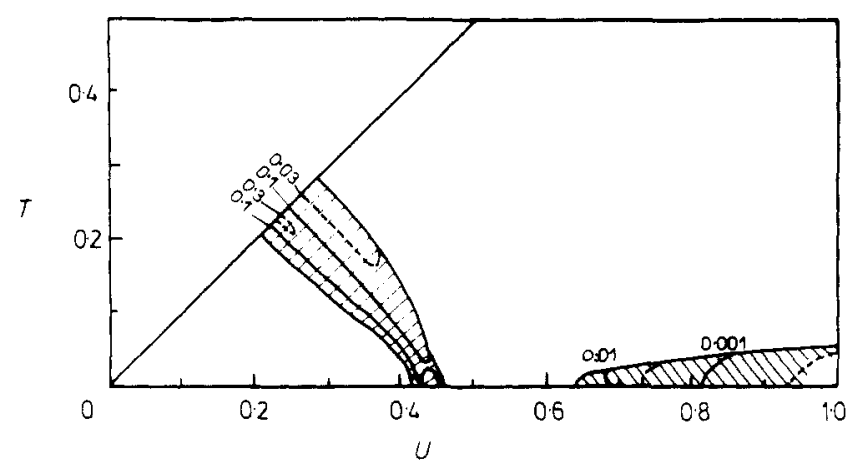

Figure 6. Reduced map of $\Delta g$ in aluminium for third-zone electrons. The magnetic field axis is along $h_{z}$.

the orientation of the field). Similarly, those of the $k_{y}=k_{z}$ plane must verify that $\Delta g_{y}=\Delta g_{z}$. In other terms, the maps with field axes $x$ and $y$ must show the same values on the $U=1$ line, and those with axes $y$ and $z$ must show the same values on the $U=T$ line. One can see in figures 1-6 that these conditions are quite well fulfilled by our calculations.

One characteristic feature of all the $g$ factor maps is the peculiar role played by the contact point between the two zones. which is $k_{x}=0.9649, k_{y}=0.4115$ and $k_{z}=0$ (in our coordinates: $U=0.4265$ and $T=0$ ). A lot of level lines converge at this point, a fact easily understood when looking at the formulae giving $\Delta g$, which include energy denominators that cancel at this point. Of course, near this point these formulae are no longer true and a special calculation concerning this is made in our appendix. However, such a treatment is needed only for a radius of approximately $2 \times 10^{-3}$ (in units of $U$ and $T$ ) around the degeneracy point, which means that our maps are true within the precision of the drawing. One can remark that there are rather large regions on the Fermi surface where the absolute value of $\Delta g$ is high (for instance larger than $0 \cdot 1$, a very high value for such a light metal). These regions are located in the $(U, T)$ plane, near the line of slope -1 passing by the degeneracy point just mentioned; they correspond to parts of the Fermi surface where the arms of the third zone are near to the volume of the second zone, so that there are small energy denominators involved. Also apparent on our maps is the high anisotropy of the $g$ factor (for different field orientations, the maps are very different) and the general sign of the $\Delta g$, which is negative for most of the electrons in the second zone and positive for most in the third zone.

Note that $g(\boldsymbol{k})$ is not a quantity directly measurable by experiments. We wish now to present some experimentally meaningful quantities that can be deduced from our calculations. In figures 7 and 8 histograms are drawn of the $\Delta g$ distribution in the second and third zones, respectively. The ordinate is proportional to the weight (as determined above) of all the Fermi surface parts having the $\Delta g$ given by the abscissa. One can remark on the considerable tails of these histograms, for the negative $\Delta g$ in the second zone and for the positive $\Delta g$ in the third zone; for instance, $6 \%$ of the total weight of the second-zone electrons have $\Delta g<-0.1$ and $26 \%$ of the third-zone electrons have $\Delta g>+0 \cdot 1$. (The relative weights of the second and third zones are $76 \%$ and $24 \%$ of the whole Fermi surface respectively. One can note here the important weight of the third-zone electrons.) These tails have considerable influence on the root 


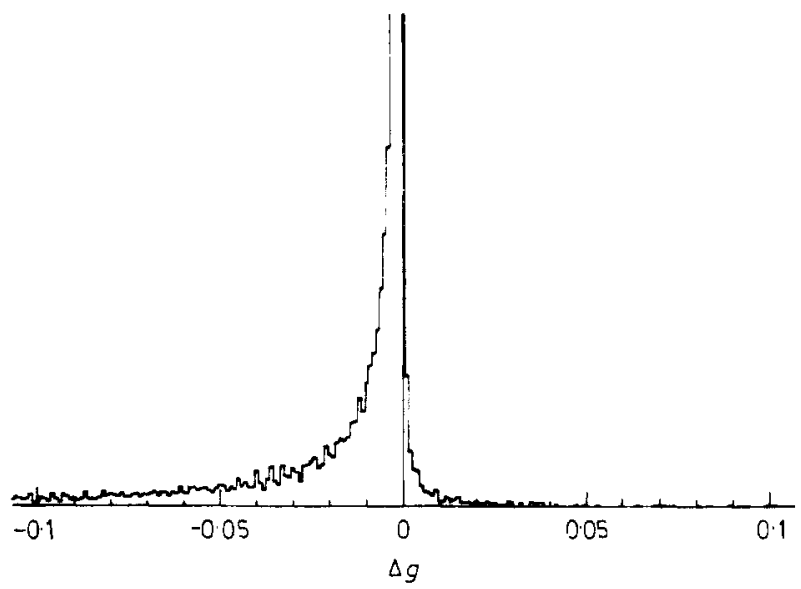

Figure 7. Histogram of $\Delta g(k)$ for each point of the Fermi surface in the second zone of aluminium. For each $\Delta g$ value in the abcissa the relative weight of the part of the Fermi surface giving this $\Delta g$ value is given in the ordinate. Note the large tail of the histogram for the negative $\Delta g$.

mean square (RMS) values of the $\Delta g$, which we give in the second column of table 1 for the two zones and for the whole Fermi surface, while in the first column we give the corresponding mean $\Delta g$ values.

These values need some comments. The lack of uniqueness of the $\Delta g$ encountered in $\$ 3$ has no influence on the mean values. On the other hand, the RMS values are not uniquely defined and one may at first suspect that their very high values are due to a non-physical artefact linked to our peculiar choice of the wavefunction phases. We believe that this is not the case, because when we consider the formula $\Delta g=\Delta g_{1}+\Delta g_{2}$, we note that only $\Delta g_{2}$ is badly defined; on the other hand, our calculations have shown that the histograms taken for $\Delta g_{1}$ and $\Delta g_{2}$ separately have RMS values to the same order of magnitude.

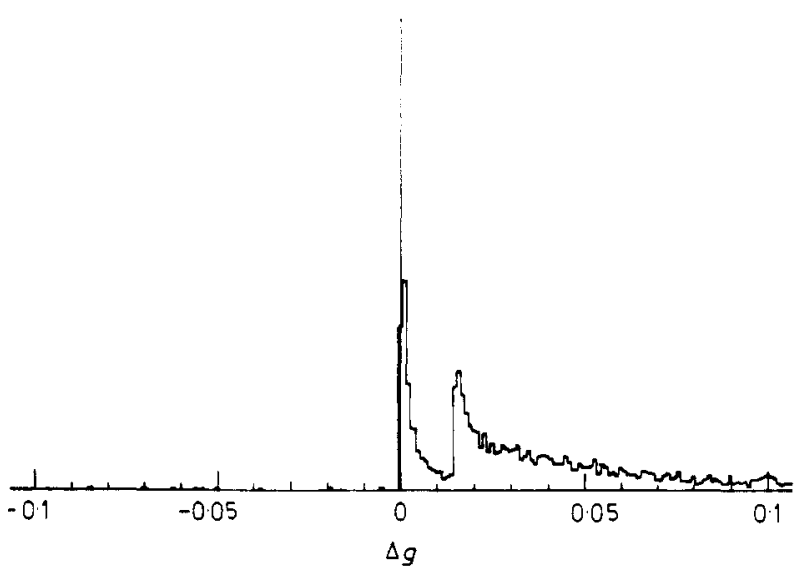

Figure 8. Histogram of $\Delta g(\boldsymbol{k})$ for each point of the Fermi surface in the third zone of aluminium. Note the large tail of the histogram for positive $\Delta g$ 
Table 1. Mean and RMS values for $\Delta g$ in aluminium.

\begin{tabular}{lccl}
\hline & & \multicolumn{2}{c}{ RMS $\Delta g$ values } \\
\cline { 3 - 4 } & Mean $\Delta g$ & $\Delta g(k)$ & $\Delta g$ of orbits \\
\hline Second zone & -0.023 & 0.272 & 0.042 \\
Third zone & 0.078 & 0.815 & 0.071 \\
Whole Fermi & 0.0013 & 0.469 & 0.067 \\
surface & & & \\
\hline
\end{tabular}

We have to comment on the mean $\Delta g$ value obtained, which is +0.001 . This value can be compared with the experimentally available $\Delta g$. For this comparison it seems coherent to consider the $g$ value obtained when one is in a motional narrowing regime, i.e. at low frequency and 'medium' temperature (see for instance Lubzens et al 1972): $g=1.996$ or $\Delta g=-0.006$.

The agreement with the calculation is very bad, as even the sign is not correctly predicted. However one must keep in mind that the total RMS value found here is 0.47 which is considerably greater than the difference between the experimental and theoretical results, making the calculated $\Delta g$ rather unprecise. Furthermore, there is a contribution to $\Delta g$ that has been forgotten so far: the formulae for $\Delta g$ are written as sums over all the energy bands and we have restricted ourselves to the four lower conduction bands as given by the four opw model. However one can estimate the extra contribution due to the deep-lying $2 \mathrm{p}$ levels of $\mathrm{Al}$. For a rough order of magnitude estimate, we take $\left|\Delta g_{2 \mathrm{p}}\right|=\lambda_{2 \mathrm{p}} / \Delta E_{2 \mathrm{p}-3 \mathrm{~s}}$ (Elliott 1954, Beuneu and Monod 1978) where $\lambda_{2 p}$ is the spin-orbit splitting of the $2 p$ states of atomic Al (see Yafet 1963) and $\Delta E$ is the energy difference between the $2 \mathrm{p}$ and $3 \mathrm{~s}$ states for atomic Al. One obtains $\left|\Delta g_{2 \mathrm{p}}\right| \approx 5 \times 10^{-3}$ which is of the order of magnitude of the difference between the experimental and theoretical $\Delta g$.

A lot of experiments in CESR in aluminium have been performed (see $\$ 1$ ) at high frequency (and magnetic field) and low temperature. One is then in a regime where the momentum relaxation rate is much lower than the cyclotron frequency (which is equal to the Larmor frequency for $g=2$ and $m^{*}=1$ ). In such a case the means of $\Delta g$ over the cyclotron orbits are physically relevant. We have considered 100 such orbits on the Fermi surface, with the magnetic field taken along $k_{z}$, varying $k_{z}$ from 0 to 1 in $2 \pi / a$ units. The mean $\Delta g$ value for a given orbit is plotted against $k_{z}$ in figures 9 and 10 , which are drawn for the second- and third-zone electrons respectively. The quasidegeneracy near $k_{z}=0.4$ is related to the previously mentioned contact point between the two zones. In figure 11 we give new $\Delta g$ histograms, which no longer concern the individual $\Delta g(k)$ values (as in figures 7 and 8 ) but the mean $\Delta g$ values over the cyclotron orbits. The striking difference between these histograms and those of figures 7 and 8 is the absence of the high $\Delta g$ tails, which implies much lower RMs values for the orbital $g$ distributions: these values are listed in the last column of table 1 . One must keep in mind that the $\Delta g$ over cyclotron orbits, and the RMS values of these $\Delta g$, are not phase dependent and thus are defined unambiguously.

Among the different cyclotron orbits, the extremum ones are particularly interesting. It is well known that only orbits with an extremum area can be observed by such techniques as the de Haas-van Alphen effect. These techniques sometimes allow the measurement of the mean $g$ factor over extremum orbits, and a lot of work has been 


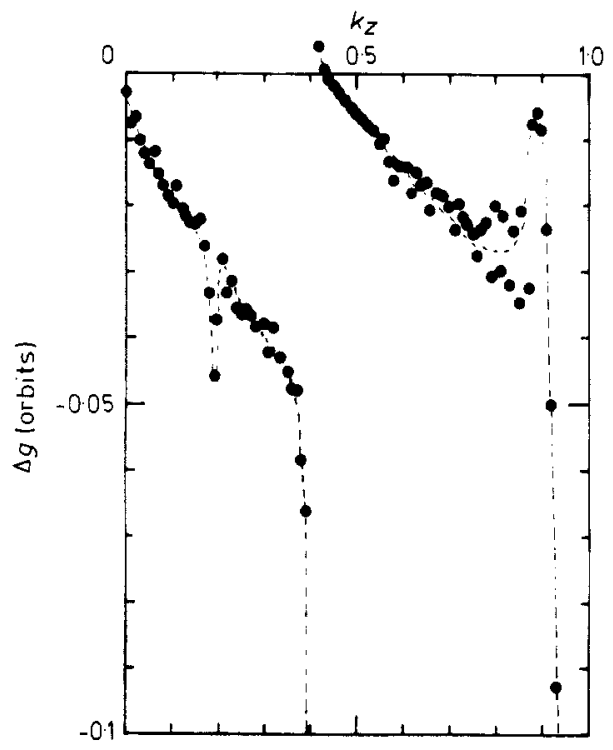

Figure 9. Mean $\Delta g$ values over cyclotron orbits versus $k_{z}$, for the second zone. The full circles are calculated values and the broken curve is just to guide the eye. The discontinuity near $k_{z}=0.4$ corresponds to the degeneracy point discussed in the appendix.

done in noble metals (see Randles 1972, Bibby and Shöenberg 1977, Crabtree et al 1977). We know of no such measurements performed in aluminium, and have found it interesting to calculate the mean $g$ value on some extremum orbits that have been studied by the de Haas-van Alphen effect. We use the notation taken from Anderson

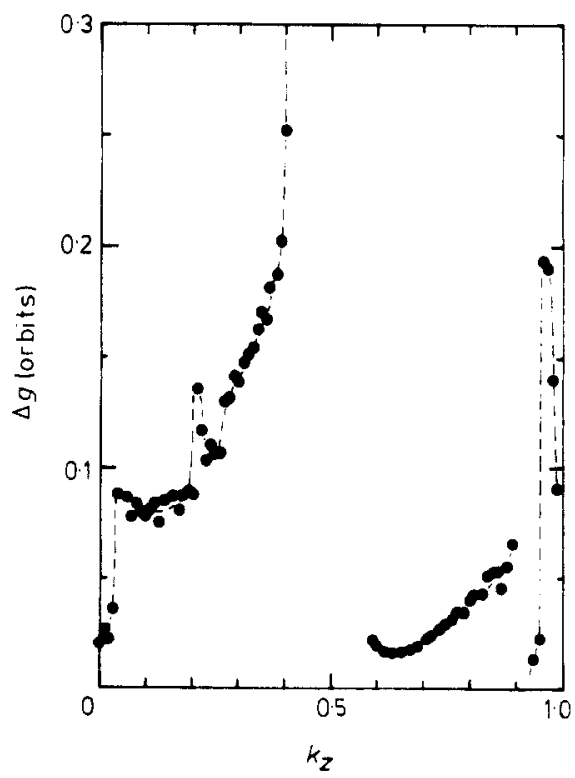

Figure 10. Mean $\Delta g$ values over cyclotron orbits versus $k_{z}$, for the third zone. Note that some $k_{z}$ values correspond to no third-zone points. The discontinuity near $k_{z}=0.4$ corresponds to the degeneracy point discussed in the appendix. 


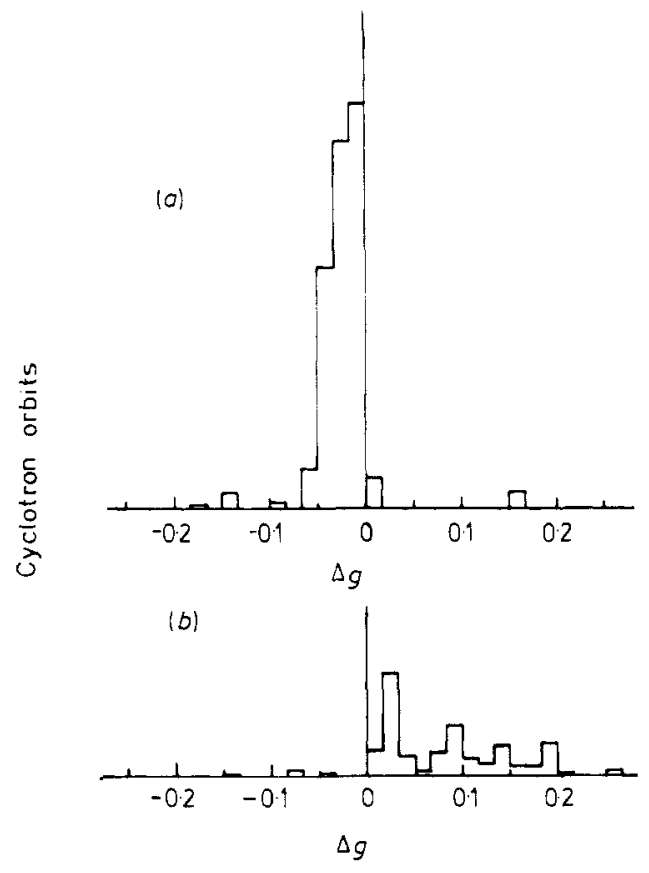

Figure 11. Histograms of the mean $\Delta g$ values over cyclotron orbits, for $(a)$ the second and (b) the third zone. The distribution tails of figures 7 and 8 are absent here.

and Lane (1970), recalled in figure 12. In table 2 we give the results of the calculations for the extremum orbits, with the corresponding field orientation.

To conclude this presentation of our calculations, we just mention that all our results are nearly unmodified by slight parameter modifications. For instance we tried the parameters proposed by Anderson and Lane (1970) which are $E_{\mathrm{F}}=0.8667$ Ryd, $V_{11^{\prime}}=0.018$ Ryd and $V_{200}=0.062 \mathrm{Ryd}$ and we obtained no significant changes in $\Delta g$ values and means.
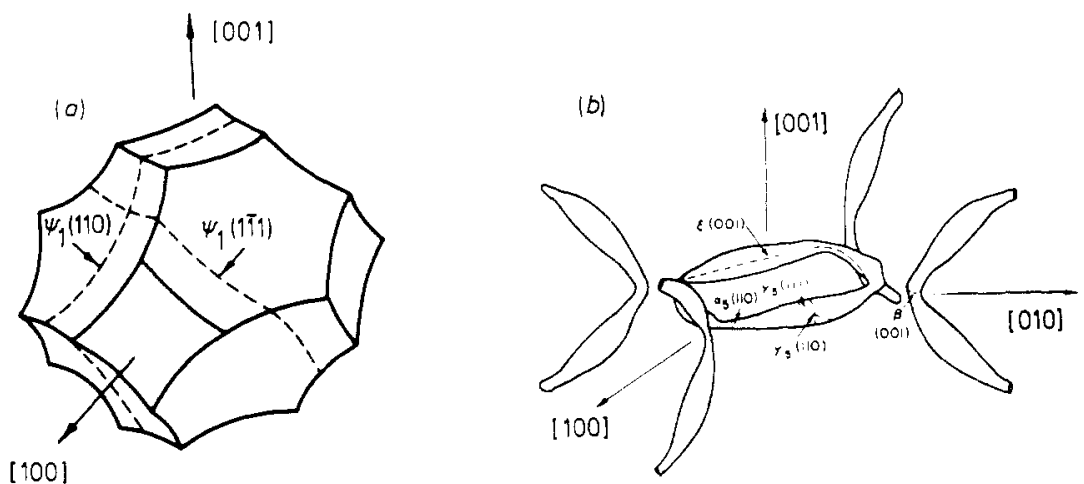

Figure 12. Main extremal orbits which can be observed in aluminium by the de Haas-van Alphen effect (Anderson and Lane 1970) in (a) the second and (b) the third zones. 
Table 2.

\begin{tabular}{lll}
\hline Orbit & Orientation & Mean $\Delta g$ \\
\hline$\psi_{1}$ & 100 & -0.0028 \\
$\psi_{1}$ & 110 & -0.0405 \\
$\psi_{1}$ & 111 & -0.0143 \\
$\beta$ & 100 & 0.0941 \\
$\xi$ & 100 & 0.0619 \\
$\gamma_{5}$ & 110 & 0.1423 \\
\hline
\end{tabular}

\section{Discussion}

We wish to return to the problem of the lack of uniqueness of the $g(\boldsymbol{k})$ value, as this question is as yet very unclear. We saw that in the absence of collisions, cyclotron orbits were well defined and the relevant $g$ factors were those of the orbits, which were unambiguous. The problem arises in the presence of collisions, as de Graaf and Overhauser (1969) pointed out, and especially when $\omega \tau \ll 1$, i.e. when it is no longer possible to consider cyclotron orbits. These authors used a wave-packet treatment, leading them to $g$ values which were independent of the wavefunction phase. Their calculation was corrected later by Moore (1975a) or, quite similarly, by Singh et al (1976); these treatments suppress all $g$ factor ambiguity coming from phase problems and would modify our $\Delta g_{2}$ term. However this semiclassical wave-packet description was criticised by Lamb (1975) who claimed that such a model did not offer a good frame for the description of the CESR phenomena. The reason for considering this model is that one wishes to use the usual motional narrowing arguments in a regime where collisions are numerous; with the spin-orbit interaction these collisions are linked to spin-flip scattering, which becomes mixed in a complex fashion with the radio-frequency spin-flipping. Lamb's opinion is that any wave-packet approach implicitly ignores this problem and cannot account for the CESR in a rigorous way. This complicated controversy means that the problem is still open. However our feeling is that individual $g(\boldsymbol{k})$ need not be well defined, as they are not physically observable quantities. One has only to propose a narrowing mechanism such that the finally observable quantity be defined uniquely. The only tentative approach in this direction is that of Lamb (1975) and is restricted to the case when $\omega \tau \gg 1$. We shall not propose any answer to the problem in the general case. We believe that a way of approaching the $\omega \tau \ll 1$ case is as follows. In a $\boldsymbol{k} \rightarrow \boldsymbol{k}^{\prime}$ collision the phaseshift is well defined and may be calculated for simple cases. This imposes one condition between the a priori arbitrary phases of the $\boldsymbol{k}$ and $\boldsymbol{k}^{\prime}$ wavefunctions. As each collision introduces such a phase relation, we hope that it is possible to avoid the ambiguity problem even in the case where the collisions are numerous $(\omega \tau \ll 1)$.

From the above considerations, it is clear that the model which we present now cannot be considered as rigorous; we will give only qualitative arguments whose purpose is to explain most of the strange CESR properties of aluminium. As we explained in $\$ 1$, we consider it obvious that aluminium electron spins are in a motional narrowing regime. A further argument can be taken from the RMS value of $g$, called $\sigma_{g}$ hereafter, that can be deduced from the slope of $\Delta H$ versus frequency, under the hypothesis of no motional narrowing: $\sigma_{g}=0.0088$. This value is considerably 
lower than the 0.067 value computed here in the cyclotron motion regime, which does not seem to be understandable. In the simple motional narrowing theory (see for instance Pines and Slichter 1955) one can write:

$$
\frac{1}{2} \Delta H=\gamma^{-1} \Delta \omega^{2} \tau=\gamma^{-1} \frac{1}{4} \sigma_{g}^{2} \omega^{2} \tau
$$

where $\tau$ is the momentum relaxation time, which will be taken as the resistivity lifetime. Here $\Delta H$ is only the part of the CESR linewidth due to $g$ factor anisotropy: the linewidth due to spin relaxation has been subtracted. We showed in $\$ 1$ why this model cannot account for the CESR properties of aluminium: the experimental law is linear with frequency and shows only slight dependence on temperature (i.e. on $\tau$ ). Our idea is that these difficulties are due to the cyclotron motion being forgotten in such a simple model, but it must cause some narrowing in a different way to a random walk motion.

We found it simple to treat this problem as follows. Starting with the simple motional narrowing formula just given above, we suppose that $\sigma_{g}$ varies with $\omega \tau$. Note that we take in this extremely simplified model a unique cyclotron frequency, supposed to be equal to the Larmor frequency $\omega$. For $\omega \tau \gg 1$, a conduction electron travels around its cyclotron orbit for a long time before being diffused, so that the $g$ factor distribution of this orbit is completely narrowed. For this regime it is then natural to take $\sigma_{g}=0.067$, computed above for the $\Delta g$ of the orbits. This is equivalent to the results obtained on a more rigorous basis by Lamb (1975). In the opposite regime, $\omega \tau \ll 1$, the cyclotron motion can be neglected because of the rapid random diffusion over the Fermi surface. We shall take $\sigma_{g}=0.47$ here which is the RMS value obtained for the individual $g(\boldsymbol{k})$. We now choose a completely arbitrary interpolation law between the two regimes, imposing $\sigma_{g}=\alpha(\omega \tau)^{-1 / 2}$ between the two extreme values for $\sigma_{g}$. In this intermediate regime we obtain:

$$
\frac{1}{2} \Delta H=\frac{\alpha^{2}}{4 \gamma} \frac{\omega^{2} \tau}{\omega \tau}=\frac{\alpha^{2}}{4 \gamma} \omega .
$$

We have chosen the law for $\sigma_{\theta}$ such that $\Delta H$ varies linearly with $\omega$. The interesting feature, which is not trivial, is the disappearing of $\tau$ : in this extremely simple model, $\Delta H$ does not vary with temperature (keeping in mind that here we forget the spin relaxation contribution adding to $\Delta H$ in practice).

In figure 13 we give the variation of the CESR linewidth of aluminium (always forgetting the phonon spin relaxation) with frequency for a wide range of scattering time $\tau$, as can be predicted by our model. The linear parts of the curves have been given a reasonable slope by fitting the parameter $\alpha$ mentioned above. One can see that for $\tau$ ranging from $3 \times 10^{-13} \mathrm{~s}$ to $10^{-11} \mathrm{~s}$ the curves obtained show an important linear part. These $\tau$ values have reasonable orders of magnitude over the $0-80 \mathrm{~K}$ temperature range. Moreover the parabolic rise above $50 \mathrm{GHz}$ shown in figure 13 by the curve $\tau=10^{-11} \mathrm{~s}$ agrees with the experimental deviation from linearity observed by Dunifer and Pattison (1976) at low temperature.

There is a complementary method for testing our model $\uparrow$. In the motional narrowing formula

$$
\frac{1}{2} \Delta H=\frac{1}{4} \gamma^{-1} \sigma_{g}^{2} \omega^{2} \tau
$$

+ We are indebted to Professor W Kohn who suggested this approach. 


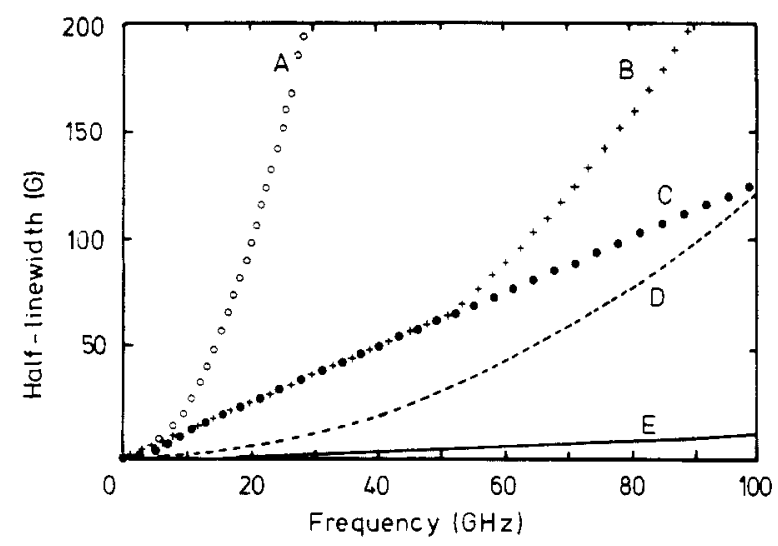

Figure 13. Predicted CESR linewidth of aluminium versus frequency. Spin relaxation has been omitted and should be added to the frequency-dependent width. Our model predicts different behaviour for different values of the scattering time $\tau$; the curves correspond to the following $\tau$ values: A, $10^{-10} \mathrm{~s} ; \mathrm{B}, 10^{-11} \mathrm{~s} ; \mathrm{C}, 10^{-12} \mathrm{~s} ; \mathrm{D}, 10^{-13} \mathrm{~s} ; \mathrm{E} .10^{-14} \mathrm{~s}$.

every quantity except $\sigma_{g}$ is accessible experimentally (with a large indeterminate $\omega$ and $\tau)$. It is thus possible to plot the experimental variation of $\sigma_{g}$ with $\omega$ and $\tau$. This is done in figure 14, taking the easily attainable parameter $F / \rho$ as a representation of $\omega \tau$ (where $F$ is the CESR frequency and $\rho$ the resistivity of the sample at different temperatures). The conclusions are that $\sigma_{g}$ varies quite like $(\omega \tau)^{-1 / 2}$, and figure 14 also shows that $\omega \tau$ is a good parameter with which to define $\sigma_{g}$; we obtain the same $\sigma_{g}$ for different $\omega$ and $\tau$ having the same $\omega \tau$ value. On the other hand, the regimes $\omega \tau \gg 1$ and $\omega \tau \ll 1$ are badly described, particularly for $\omega \tau \ll 1$ where $\sigma_{g}$ exceeds the calculated maximum value $\left(\sigma_{g}=0.47\right)$. We note that this regime corresponds to the hightemperature range when the spin-flip due to phonons makes data analysis particularly difficult.

It is interesting to note that Fredkin and Freedman (1972) have considered, as we did here, the influence of the cyclotron motion on the linewidth, which is expressed by their formula (4). However they probably considered the effect to be unimportant because they were not aware of the large difference between the two extreme values of $\sigma_{g}$ found by us. The simplest form of their formula (4) gives:

$$
\sigma_{\theta}=\left(G_{1}+\frac{G_{2}}{1+\omega^{2} \tau^{2}}\right)^{1 / 2}
$$

This expression for $\sigma_{g}$ has qualitatively the same behaviour as the empirical one used above: $\sigma_{g}$ is constant for very high and very low values of $\omega \tau$, and there is an intermediate regime where $\sigma_{g}$ decreases with increasing $\omega \tau$. However such a law will not ensure a linear $\Delta H$ versus frequency law. However one must keep in mind that $\tau$ is a very badly defined quantity. Wegehaupt and Doezema (1978) measured a very high anisotropy of $\tau$ over the Fermi surface, and this complication made any comparison difficult between the simple formulae obtained with the hypothesis that $\tau$ is isotropic, and experiment.

We have not discussed so far the experimental variation of $g$ with temperature and 


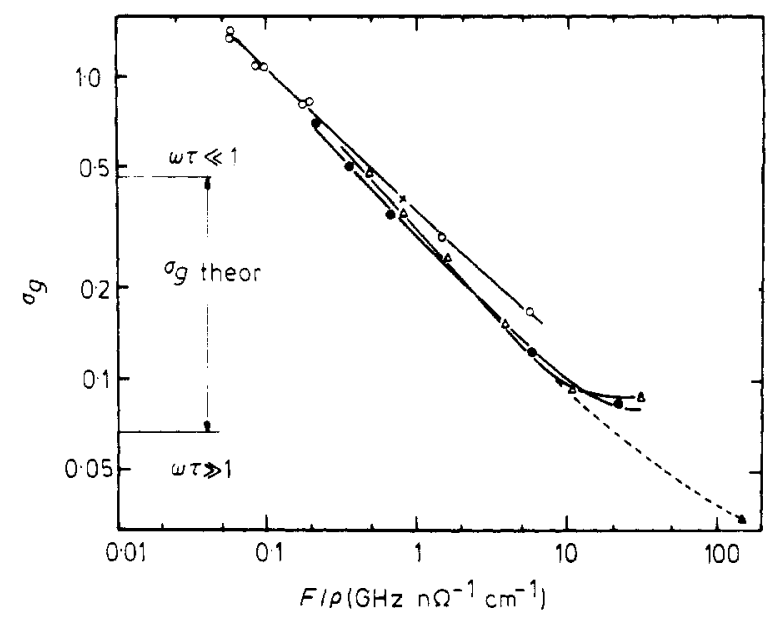

Figure 14. $\sigma_{g}$ values taken from experiment and with the assumption of simple motional narrowing. versus the ratio of frequency to resistivity. This ratio approximately describes the product $\omega \tau$. The curves relate the points corresponding to a given sample measured at a given frequency $(\times .1 .27 \mathrm{GHz}: 0,9 \cdot 2 \mathrm{GHz} ; 0.35 \mathrm{GHz}, \triangle, 79 \mathrm{GHz}(27 \mu \mathrm{m}) ; \Delta, 79 \mathrm{GHz}$ $(132 \mu \mathrm{m}))$ and at different temperatures. The theoretical $\sigma_{g}$ values in the two limits $\omega \tau \gg 1$ (narrowing by orbital motion) and $\omega \tau \ll 1$ (narrowing by collisions) are included.

frequency in aluminium. In principle, it is not necessary to invoke Fermi liquid effects to account for this variation, because the $g$ factor distribution is not symmetric around its mean. However we shall show that these effects, described by Fredkin and Freedman (1972), give a good order of magnitude for the $g$ shifts. The authors find that

$$
\Delta g=\frac{X^{2}}{1+X^{2}} \frac{(1+B)^{2} \sigma_{g}^{2}}{2 B} \quad \text { with } X=\frac{B \omega \tau}{1+B} .
$$

Again imposing $\sigma_{g}=\alpha(\omega \tau)^{-1 \cdot 2}$ and taking $X^{2} \ll 1$ and $B \ll 1$ we obtain

$$
\Delta g=\frac{1}{2} B \alpha^{2} \omega \tau
$$

Dunifer et al (1977) determined $B=+0.1$ by observing spin waves in aluminium. For $\tau=2 \times 10^{-11} \mathrm{~s}$ we find $\Delta g=0.004$ at $35 \mathrm{GHz}$ and 0.010 at $79 \mathrm{GHz}$; for samples of resistivity giving such a $\tau$ value, one has $\Delta g=0.009$ at $35 \mathrm{GHz}$ and 0.012 at $79 \mathrm{GHz}$ (Lubzens and Schultz 1976, Dunifer and Pattison 1976). The agreement is qualitatively correct at both frequencies, although non-qualitatively correct at $35 \mathrm{GHz}$.

In conclusion, we believe that the idea of a double type of motional narrowing (from a coherent and a random motion) is the relevant one for understanding the strange CESR properties of aluminium. We obtained, with very simplified models, qualitative agreement with experiments. We think that it would be necessary to take into account the anisotropy of $\tau$ (and of the cyclotron frequency) in order to obtain quantitative agreement. However our model is unable to explain the anomalous value of the static susceptibility of aluminium. On the other hand we hope that the mechanism of narrowing described here will help to understand the CESR properties of other complex metals. 


\section{Acknowledgments}

The author wishes to thank Professor $\mathbf{J}$ Friedel and Dr P Monod for numerous discussions and constant guidance throughout this work. Discussions with Professor W Kohn, Professor M Bloom, Dr Y Quéré, Dr Y Yafet and Dr G L Dunifer are gratefully acknowledged. The author is indebted to Dr G L Dunifer for communicating his ESR results on copper before publication.

\section{Appendix. Treatment of the point of degeneracy}

The point of reciprocal space with coordinates $k_{x}=0.9649, k_{y}=0.4115$ and $k_{z}=0$ is a point where two energy bands intercept at the Fermi level. As the spin-orbit lifts the degeneracy at this point, it cannot be treated by the perturbation scheme developed in $\S 2$. We wish to give here, in the vicinity of this contact point between the second and third zones, some indications of the treatment leading to the $g$ factor. For a detailed account, see Beuneu (1979).

The part of the Fermi surface for which the general calculation of $\$ 2$ is no longer valid is quite small; it corresponds to those points where the energy gap is smaller than the spin-orbit splitting of the $3 p$ states, which is approximately $10^{-3} \mathrm{Ryd}$. In such a region we can neglect the two bands (obtained in the four OPW model) which are not in the vicinity of the Fermi level. Let $\psi_{1}$ and $\psi_{2}$ be the nearly degenerate wavefunctions calculated using the Ashcroft model (without the spin-orbit). Introducing the spin-orbit leads to a secular equation which is a $4 \times 4$ determinant, with basis functions $\psi_{1} \uparrow, \psi_{2} \uparrow, \psi_{1} \downarrow$ and $\psi_{2} \downarrow$.

From this equation one can determine the wavefunctions (with the spin-orbit) and apply the $\Delta g$ formulae given in $\$ 3$. Let $\Delta E$ be the energy splitting due to the spinorbit; its order of magnitude is $10^{-3}$ Ryd. Defining

$$
\text { i } A=\frac{1}{2}\left\langle\Psi_{1}\left|\lambda(r) l_{z}\right| \Psi_{2}\right\rangle
$$

with $A$ real we get for $\Delta g_{1}$ :

$$
\Delta g_{1} \simeq \frac{4}{m} \frac{A}{(\Delta E)^{2}}\left[\left\langle\psi_{1}\left|p_{x}\right| \psi_{2}\right\rangle\left(\left\langle\psi_{1}\left|p_{y}\right| \psi_{1}\right\rangle-\left\langle\psi_{2}\left|p_{y}\right| \psi_{2}\right\rangle\right)-x \rightleftarrows y\right] .
$$

The formula for $\Delta g_{2}$ is more complicated and will not be reproduced here.

We wish here only to estimate the order of magnitude of $\Delta g$ around the contact point. Taking $\Delta E \simeq 10^{-3}$ Ryd near this point and considering all the matrix elements of $p$ to be of the order of unity, we obtain a value of a few hundred for $\Delta g$. On the other side we have calculated the relative weight of those points on the Fermi surface for which $\left|E\left(\Psi_{1}\right)-E\left(\Psi_{2}\right)\right|<10^{-3}$ Ryd and found nearly $5 \times 10^{-6}$ for each zone. The variation of the mean $\Delta g$ due to the degeneracy point is thus of the order of $10^{-3}$; furthermore it should be noted that the signs of $\Delta g$ are opposite for the two zones.

\section{References}


Asik J R, Ball M A and Slichter C P 1969 Phys. Rev. 181 645-61

Beuneu F 1979 PhD Thesis Orsay University. France (Rapport CEA R-5026)

Beuneu F and Monod P 1978 Phys. Ret: B 18 2422-5

Bibby W M and Shöenberg D 1977 Phys. Lett. 60A 235-6

Bienenstock A and Brooks H 1964 Phys. Ret. 136 A784-802

Blount E I 1962 Phys. Rer. 126 1636-53

Braim S P. Sambles J R, Cousins J E, Van Meijel J, Stesmans A and Witters J 1979 Solid St. Comun. 29 $621-4$

Cohen M H and Blount E I 1960 Phil. Mag. 5 115-26

Crabtree G W. Windmiller L R and Ketterson J B 1977 J. Low. Temp. Phys. 26 755-62

Delafond J, Junqua A and Mimault J 1973 Phys. Stat. Solidi 15 553-8

Dunifer G L 1980 to be published

Dunifer G L and Pattison M R 1976 Phys. Rev. B $14945-50$

Dunifer G L. Pattison M R and Hsu T M 1977 Phys. Rer. B 15 315-22

Elliott R J 1954 Phys. Rer. 96 266-79

Fredkin D R and Freedman R 1972 Phys. Ret. Lett. $291390-3$

Freediman R and Fredkin D R 1975 Phys. Rev. B 11 4847-58

de Graaf A M and Overhauser A W 1969 Phys. Rev. 180 701-6

Harrison W A 1966 Pseudo-potentials in the Theory of Metals (New York: Benjamin)

Hartree D R 1935 Proc. R. Soc. A 151 96-105

Janssens L, Stesmans A, Cousins J E and Witters J 1975 Phys. Stat. Solidi b 67 231-7

Kohn W 1959 Phys. Rev. 115 1460-78

Lamb W J 1975 PhD Thesis University of California

Lenglart P 1967 J. Phy's. Chem. Solids $282011-25$

Lubzens D and Schultz S 1976 Phys. Rer. Lett. 36 1104-6

Lubzens D. Shanabarger M R and Schultz S 1972 Phys. Ret. Lett. 29 1387-90

Mimault J, Delafond J and Junqua A 1973 Phys. Stat. Solidi 20 195-200

Monod P and Beuneu F 1979 Phys. Ret. B 19 911-6

Moore R A 1975a J. Phy's. F: Metal Phys. 5 459-67

- - 1975b J. Phys. F: Metal Phi's. 5 2300-6

Pines D and Slichter C P 1955 Phys. Ret: $1001014-20$

Rahman T S, Parlebas J C and Mills D L 1978 J. Phys. F: Metal Phys. 8 2511-24

Randles D L 1972 Proc. R. Soc. A 331 85-101

Roth L M 1960 Phy's. Rer. 118153440

- 1962 J. Phys. Chem. Solids 23 433-46

Sambles J R, Cousins J E. Stesmans A and Witters J 1977a Solid St. Commun. 24 673-6

Sambles J R, Sharp-Dent G. Cousins J E, Stesmans A and Witters J 1977b Phys. Stat. Solidi b 79 645-54

Sharp-Dent G. Hardiman M. Sambles J R and Cousins J E 1976 Phys. Stat. Solidi b 75 155-61

Singh M. Callaway J and Wang C S 1976 Phys. Ret. B 14 1214-20

Stesmans A. Van Meijel J and Braim S P 1979 Phrs. Rei. B $195470-4$

Van Meijel J. Stesmans A and Witters J 1977 Solid. St. Commun. 21 753-5

Wegehaupt T and Doezema R E 1978 Phys. Rer. B $18742-8$

Wilson A H 1953 The Theory of Metals (London: Cambridge University Press)

Yafet Y 1957 Phy's. Ret. 106 679-84

-1963 Solid St. Phis. 14 1-98 